Ensino, Saúde e Ambiente - V6 (1), pp.89-100,abr. 2013

\title{
PRÁTICA PEDAGÓGICA DIFERENCIADA NOS PROCESSOS DE ENSINAR E DE APRENDER EM PARASITOLOGIA
}

\section{DIFFERENTIATED IN EDUCATIONAL PRACTICE PROCEDURES IN TEACHING AND LEARNING PARASITOLOGY}

\author{
Camila Grimes, Daiane Luchetta Ronchi e Zelinda Maria Braga Hirano \\ Universidade Regional de Blumenau - FURB \\ Rua Antônio da Veiga, 140, Bairro Victor Konder, \\ Blumenau-SC CEP 89012-900 \\ camilagrimes@ibest.com.br \\ daianeronchi@hotmail.com \\ zehirano@hotmail.com
}

\begin{abstract}
RESUMO
O ensino de Saúde representa um grande desafio para a educação, pois tem como função garantir uma aprendizagem que promova mudança de atitudes e hábitos. As intervenções educativas podem instigar ações que colaborem para prevenção de parasitoses. Assim, o presente estudo teve como objetivo, avaliar o uso de estratégias educativas diferenciadas no ensino e na aprendizagem de Parasitologia. O trabalho foi realizado com base no Projeto Painel Itinerante - Parasitologia, desenvolvido pelos bolsistas do PET/Biologia/FURB. Para a coleta de dados utilizou-se a estratégia estudos de caso, na qual foram contabilizados os parasitos identificados pelos estudantes do Ensino Fundamental, de escolas públicas, em Blumenau (SC). Os resultados obtidos na atividade estudos de caso indicam que os estudantes identificaram $71 \%$ dos casos analisados. O projeto mostrou-se uma importante atividade na educação em saúde, pois a prática poderá influenciar no ambiente social dos estudantes, visto que eles poderão tornar-se divulgadores do conhecimento científico.
\end{abstract}

Palavras-chave: Parasitologia, Educação em Saúde, Prática pedagógica, Processos de Aprender, Ensino de Ciências.

\begin{abstract}
Health education is a major challenge for education, because its function is to ensure that learning promotes changing in attitudes and habits. Educational interventions can instigate actions that cooperate to prevent parasitosis. Thus, the present study aimed to evaluate the use of different educational strategies in Parasitology teaching and learning. The study was conducted based on the Project Itinerant Panel - Parasitology, developed by scholars from PET/Biology/FURB. To collect the data we used the strategy of case study, in which the parasites were identified by students from elementary school from cases they were exposed to. The students belonged to the schools in Blumenau (SC). The results obtained in the activity case study indicated that students identify $71 \%$ of the analyzed cases. The project proved to be an important activity in health education, because the practice can influence the social environment students, since they may become disseminators of scientific knowledge.
\end{abstract}

Keywords: Parasitology, Health Education, Educational Practice, Learning Processes, Science Teaching. 


\section{Introdução}

As parasitoses intestinais estão amplamente distribuídas no globo terrestre. $\mathrm{O}$ Brasil apresenta altas taxas de prevalência em algumas regiões (PEDROSO; SIQUEIRA, 1997). Toscani et al. (2007) destacam os principais causadores das parasitoses intestinais, e bem como, seus ciclos de vida:

As parasitoses intestinais são infecções causadas por protozoários (Giardia lamblia e Entamoeba histolytica), platelmintos (Taenia solium, Taenia saginata e Hymenolepis nana) e nematódios (Trichuris trichiura, Strongyloides stercolaris, Enterobius vermicularis, Ascaris lumbricoides, Ancylostoma duodenale e Necator americanus). Esses agentes etiológicos apresentam ciclos evolutivos que contam com períodos de parasitose humana, períodos de vida livre no ambiente e períodos de parasitose em outros animais.

Estas parasitoses representam um grave problema de saúde pública, contribuindo, muitas vezes, para problemas econômicos e sociais. Deste modo, as condições higiênico-sanitárias do ambiente em que as pessoas vivem, muitas vezes, podem contribuir para uma maior prevalência de parasitoses intestinais (ZAIDEN et al., 2008).

Segundo Costa-Macedo e Rey (2000), as crianças apresentam maior ocorrência de infecções parasitárias, sendo que no Brasil, mais da metade de pré-escolares e escolares estão parasitados. Neste contexto Ikegami et al. (1999) inferem que as crianças são mais suscetíveis às infecções parasitárias do que os adultos, pois, muitas vezes, se contaminam por não terem o conhecimento adequado das práticas de profilaxia. Assim, a infecção por meio da via oral-fecal é a mais comum, e a água e os alimentos contaminados são os principais veículos de transmissão (TOSCANI et al., 2007).

Diante disso, a educação em saúde no controle das parasitoses é de suma importância, pois é uma estratégia de baixo custo capaz de atingir resultados significativos e duradouros (ASAOLU; OFOEZIE, 2003).

Segundo os Parâmetros Curriculares Nacionais, Temas Transversais - Saúde, o ensino de Saúde é um desafio para a educação, no âmbito de garantir uma aprendizagem significativa e transformadora de atitudes e hábitos de vida. Deste modo, na educação para a saúde leva-se em conta a formação de hábitos e atitudes desenvolvidos no ambiente escolar (BRASIL, 1998).

Complementado esse pensamento, nos Parâmetros Curriculares Nacionais, no ensino de Ciências Naturais, a saúde é apresentada como um estado de equilíbrio do 
corpo e um bem da coletividade. A abordagem do conteúdo pode ser feita de maneira simples e diversificada, sendo que os nomes das doenças, seus agentes e sintomas são conteúdos desenvolvidos em atividades significativas para os estudantes, de acordo com o contexto que estão inseridos (BRASIL, 1998). Assim, nos processos de aprendizagem o professor tem importante papel na mediação dos conteúdos, visto que os estudantes trazem consigo conhecimentos espontâneos, adquiridos ao longo de sua vida, deste modo, os processos interativos entre professor-estudante e estudante-estudante são fundamentais na construção do conhecimento (COLL, 1994).

Neste sentido, as intervenções educativas podem estimular ações que contribuam para a prevenção de infecções parasitárias (TOSCANI et al., 2007). Desta forma, a aprendizagem por meio de atividades diferenciadas, pode desenvolver atitudes de prevenção, à medida que estas ações estejam associadas a políticas socioeconômicas e ambientais. Tendo essas considerações em vista, o presente estudo teve como objetivo, avaliar o uso de estratégias educativas diferenciadas no ensino e na aprendizagem de Parasitologia.

\section{O Currículo de Ciências e Parasitologia}

Nos Parâmetros Curriculares Nacionais, Ciências Naturais para o Ensino Fundamental, a temática Saúde é abordada no eixo temático Ser Humano e Saúde. Esta temática aborda as relações entre os problemas de saúde e fatores econômicos, políticos, sociais e históricos. Tais problemas ocasionam debates sobre responsabilidades humanas voltadas ao bem-estar de todos. Sendo realizada na disciplina de Ciências, a discussão destas problemáticas (BRASIL, 1998). Sugere-se ainda, nos Parâmetros Curriculares Nacionais, o desenvolvimento da seguinte capacidade relacionada ao ensino de saúde:

Compreensão do organismo humano como um todo, interpretando diferentes relações e correlações entre sistemas, órgãos, tecidos em geral, reconhecendo fatores internos e externos ao corpo que concorrem na manutenção do equilíbrio, as manifestações e os modos de prevenção de doenças comuns em sua comunidade e o papel da sociedade humana na preservação da saúde coletiva e individual (BRASIL, 1998).

De acordo com os Parâmetros Curriculares Nacionais, Temas Transversais Saúde, a escola possui um papel decisivo na construção de condutas, no qual a comunidade escolar constrói atitudes e valores no convívio cotidiano, por isso, a escola precisa assumir a responsabilidade pela educação em saúde. Neste sentido, quando os 
conteúdos referentes a saúde e doença não estão inclusos no currículo, situações cotidianas não são desenvolvidas, os estudantes convivem com infraestruturas precárias e o entorno escolar não oferecem referências saudáveis, a escola não promove cidadania, ou seja, afasta os estudantes da discussão e da prática de ações individuais e coletivas de cuidados em saúde (BRASIL, 1998).

Na Proposta Curricular do estado de Santa Catarina, em Ciências, a educação em saúde está contemplada na temática Animais/Homem, deste modo, sugere-se a abordagem de fatores que estão relacionados com as doenças dos animais, como por exemplo: tuberculose, leptospirose, raiva, fasciolose, neuro-cisticercose, toxoplasmose, dentre outras zoonoses. Além disso, estão incluídos os elementos abióticos, ar, água, solo, relacionados a saúde e bem estar. Bem como, as noções de sistemática dos reinos monera, protista, fungi, animalia e plantae relacionados ao homem. Por fim, aborda os procedimentos destinados à higiene, saúde, preservação e prevenção relacionados aos animais (SANTA CATARINA, 1998).

A educação em saúde está contemplada nos currículos, contudo, o ensino de parasitologia encontra-se fragmentado na Grade Curricular de Ciências no Ensino Fundamental, assim, muitas vezes, é pouco aprofundado ou deixa de ser abordado no ensino de Ciências.

\section{Metodologia}

O trabalho foi realizado com base no Projeto Painel Itinerante - Parasitologia, desenvolvido pelos bolsistas do Programa de Educação Tutorial (MEC) PET/Biologia/FURB. Participaram da pesquisa estudantes do $6^{\circ}$ ao $9^{\circ}$ ano do Ensino Fundamental, de escolas da rede Municipal e Estadual, em Blumenau (SC).

O Painel Itinerante leva às escolas assuntos contemporâneos, que não são abordados ou que necessitam ser complementados em sala de aula. As apresentações foram realizadas nas escolas pelos bolsistas do Programa. Durante as apresentações do Painel exibia-se um vídeo didático sobre o tema Parasitologia, e mostravam-se exemplares das espécies parasitárias abordadas (FIGURA 1). Além disso, desenvolviase uma dinâmica, na qual, os estudantes recebiam pequenos estudos de caso relacionados às parasitoses (FIGURA 2), analisavam estes casos individualmente e comparavam com as informações expostas pelos bolsistas, identificando assim, qual o parasito causador da enfermidade em questão. A coleta de dados baseou-se nesta atividade dinâmica. 


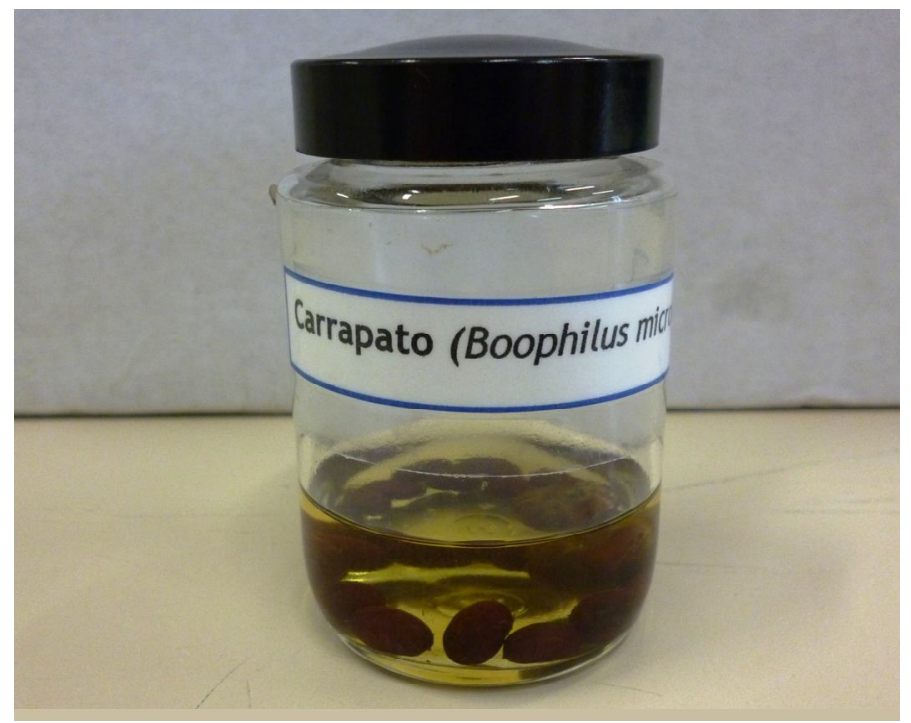

Figura 1 - Exemplar de ectoparasito utilizado nas apresentações do Painel Itinerante Parasitologia, em escolas municipais e estaduais de Blumenau (SC). Coleção Zoológica do PET/Biologia/FURB.

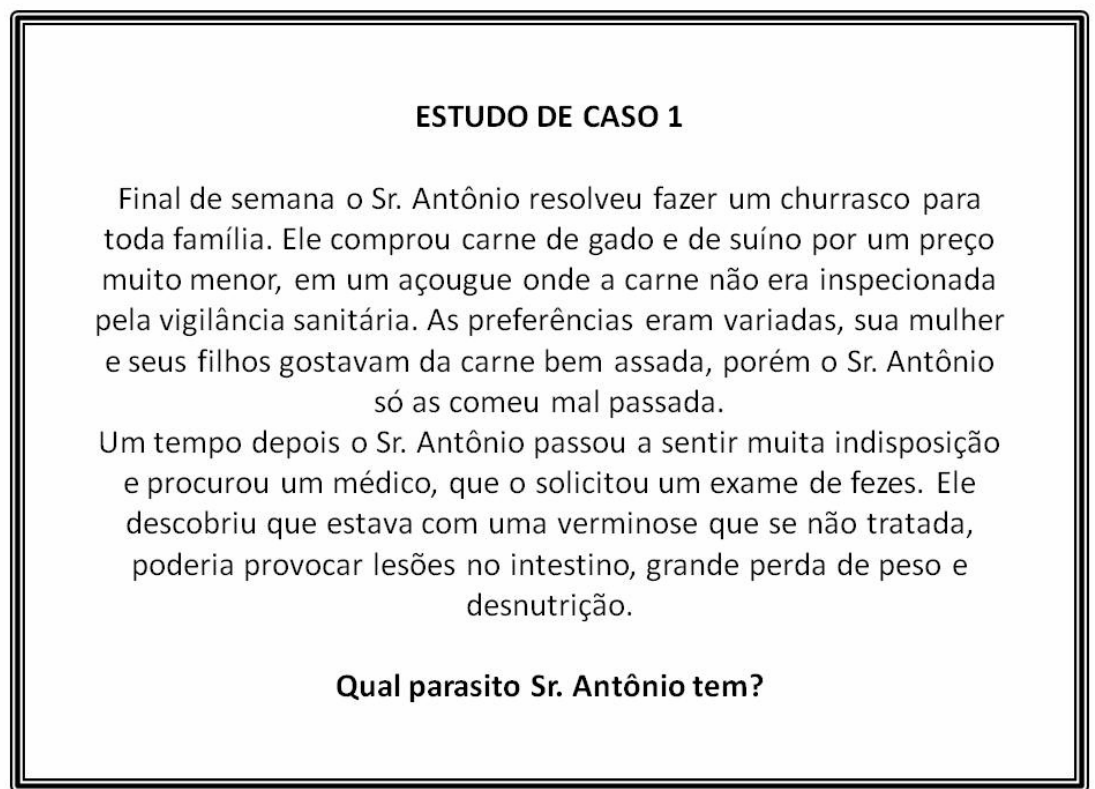

Figura 2 - Estudos de caso abordados no Painel Itinerante - Parasitologia, em escolas municipais e estaduais de Blumenau (SC).

Os parasitos abordados no Projeto Painel Itinerante Parasitologia foram: Giardia lamblia, Entamoeba histolytica, Toxoplasma gondii, Taenia solium, $T$. sarginata, Ascaris lumbricoides, Leishmania braziliensis, Schistosoma mansoni, Trypanosoma cruzi, Dermatobia hominis, Cochliomyia hominivorax, Tunga penetrans, Xenopsylla cheopis, Pediculus capitis e Amblyomma cajennense. Nestes painéis encontravam-se informações a respeito do ciclo biológico, epidemiologia, profilaxia, 
sintomas e curiosidades de cada parasito, conforme o exemplo de um dos painéis (FIGURA 3).

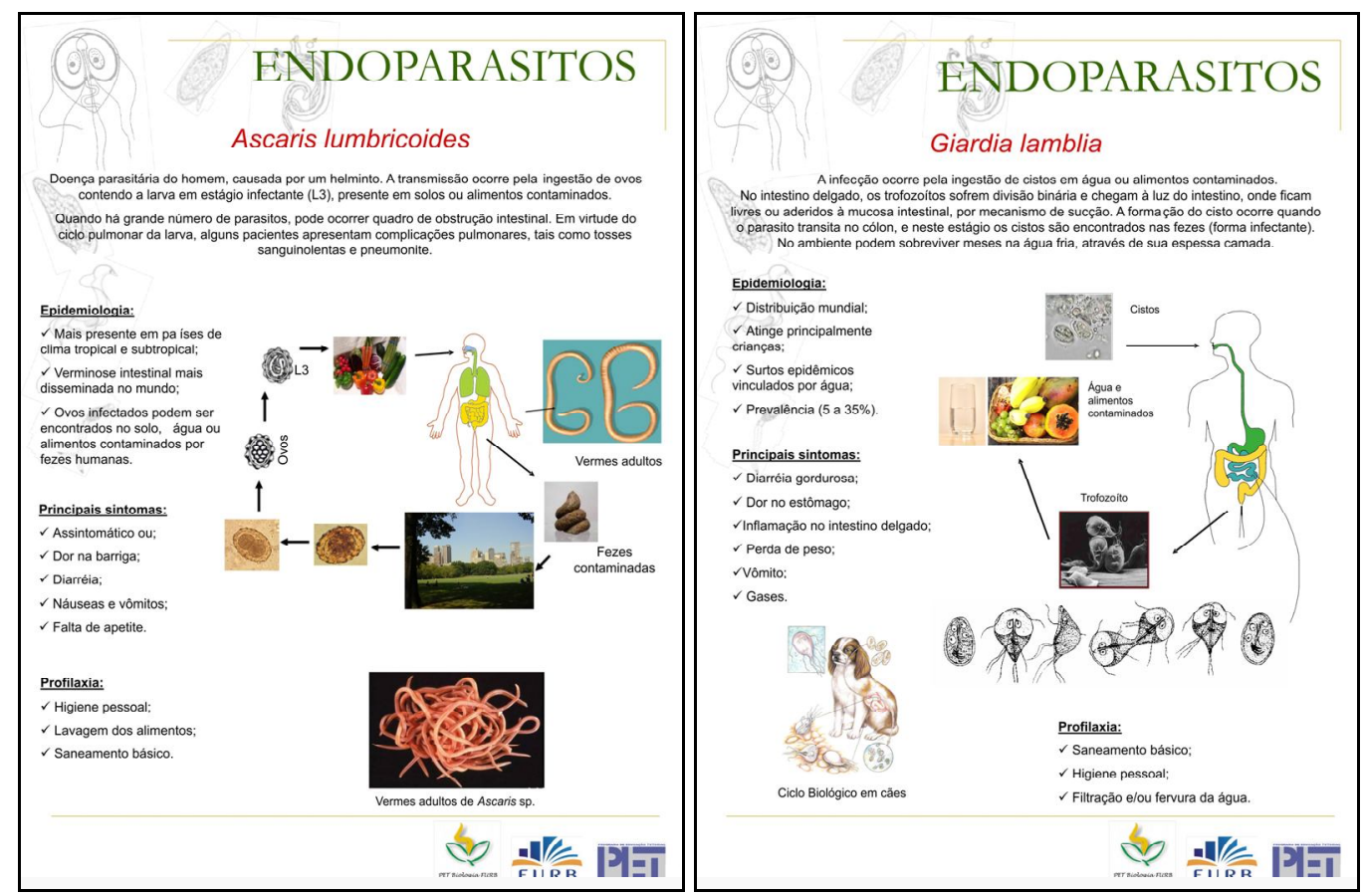

Figura 3 - Exemplares de dois painéis, Ascaris lumbricoides e Giardia lamblia, do Projeto Painel Itinerante - Parasitologia, apresentados em escolas municipais e estaduais de Blumenau (SC).

\section{Resultados e Discussão}

O projeto Painel Itinerante - Parasitologia proporcionou aos estudantes, das escolas de Blumenau e região, a aprendizagem do conhecimento científico por meio de uma atividade dinâmica e diferenciada. Atendeu aproximadamente 962 estudantes. Um dos pilares desta atividade foi a identificação dos estudos de caso de parasitologia pelos estudantes. Estes identificaram 71\% (2053 acertos) dos casos analisados. O sucesso desta atividade, possivelmente, deve-se a motivação e ao estímulo que a mesma exerce sobre os estudantes, pois esta prática pedagógica despertou a atenção, proporcionou o desenvolvimento do raciocínio e da aprendizagem, ao contrário das aulas tradicionais habituais do seu ambiente escolar. Deste modo, o docente possui o desafio e a necessidade de desenvolver práticas educativas diferenciadas e, bem como, repensar suas práticas. Pois, os recursos didáticos e estratégias de ensino, incorporados a outros fatores podem possibilitar o desenvolvimento da aprendizagem.

Neste sentido, Vasconcellos (2005) destaca que, em sala de aula, a problematização, o diálogo, a pesquisa, a experimentação, debates, jogos, dramatização, 
produção coletiva, filmes, dentre outros, são metodologias, que podem auxiliar significativamente, o processo de construção do conhecimento pelos estudantes. Ao encontro deste pensamento, Pozo (2002, p. 151) ressalta que "a diversificação das tarefas e situações de aprendizagem [...] é uma das condições mais eficazes para ativar outros processos auxiliares de aprendizagem, como a recuperação e transferência do aprendido". Vasconcellos (2005) discute ainda que, estratégias de ensino diversificadas resultam em aulas mais dinâmicas e estimulantes, sendo que estas aliadas a situações contextos promovem a construção do conhecimento. Diante disso, a situação contexto é uma das estratégias de ensino utilizadas no Painel Itinerante, na qual os bolsistas sempre buscam mediar as práticas vivenciadas pelos estudantes com os conteúdos de Parasitologia, assim, as situações contextos aproximam os conhecimentos espontâneos, realidade dos estudantes, com os conhecimentos científicos, conteúdos do ambiente escolar. Deste modo, segundo Delizoicov, Angotti e Pernambuco (2002) cabe ao professor mediar e criar situações para facilitar o aprendizado, que é construído entre as interações dos sujeitos.

$\mathrm{Na}$ estratégia estudo de caso, foi realizado o ensino por problematização, no qual os estudantes tinham que associar o parasito com o problema indicado. A problematização é uma importante estratégia nos processos de ensinar, neste sentido, Delizoicov, Angotti e Pernambuco (2002), ressaltam que o diálogo entre professor e estudante por meio da problematização, possibilita apreensão dos significados e a interpretação dos conteúdos. Nesta linha de raciocínio, Pozo (2002) infere que os estudantes necessitam se defrontar com situações-problemas ao contrário de realizar exercícios repetitivos, possibilitando assim, a tomada de decisões para a resolução de atividades.

Os parasitos identificados com maior facilidade pelos estudantes, nesta estratégia foram Toxoplasma gondii (512 acertos - 78\%), Ascaris lumbricoides (449 acertos - 77\%), Trypanosoma cruzi (539 acertos - 76\%), Schistossoma mansoni (155 acertos - 72\%) e Leishmania brasiliensis (112 acertos - 71\%). No entanto, os parasitos Giardia lamblia (157 acertos - 51\%) e Entamoeba histolytica (129 acertos - 51\%) apresentaram porcentagem de acertos semelhantes, conforme Gráfico 1. Inferimos que isso pode ter ocorrido devido ao ciclo de vida destes parasitos serem semelhantes e provocarem dificuldade de diferenciação. 


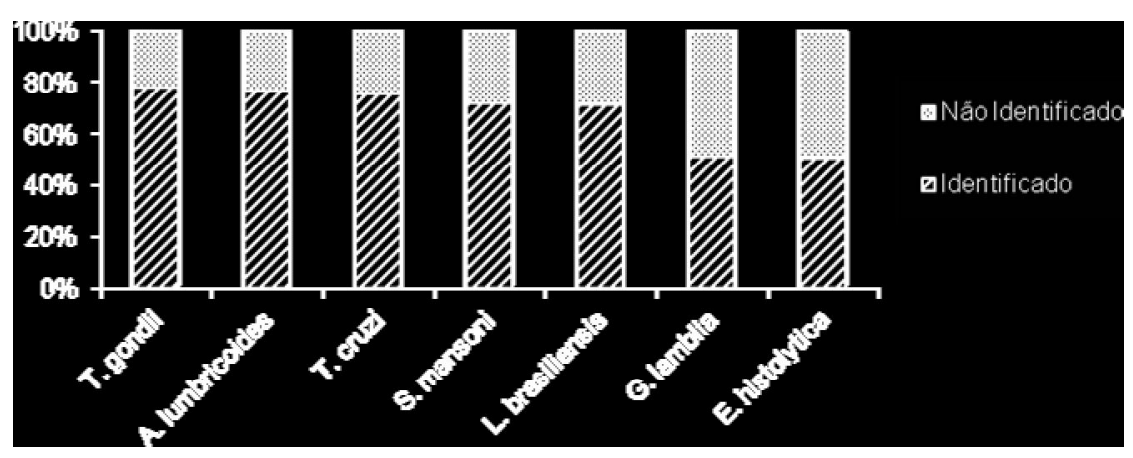

Gráfico 1 - Frequências da identificação dos parasitos, pelos estudantes de escolas municipais e estaduais de Blumenau (SC), na atividade estudo de caso, do Painel Itinerante - Parasitologia.

Os estudantes, na maioria das vezes, identificaram os estudos de caso com facilidade, demonstrando que compreenderam os conteúdos abordados no Painel Itinerante - Parasitologia, ressaltando deste modo, a relevância desta prática pedagógica. Neste sentido, Ferreira e Andrade (2005) destacam que as práticas educacionais quando bem aplicadas levam as pessoas a adquirirem os conhecimentos para prevenção de parasitoses, alcançando objetivos propostos e evidenciando o valor da orientação pedagógica para a conscientização da população. Os currículos do Ensino Fundamental estão de acordo com este pensamento, pois segundo os Parâmetros Curriculares Nacionais, Temas Transversais - Saúde, no processo de ensino, espera-se que os estudantes possam aprender e exercitar comportamentos e hábitos saudáveis, tornando-se capazes de realizar mudanças em sua vida pessoal e no ambiente em que vivem. Deste modo, a utilização de metodologias auxilia o estudante a identificar problemas, refletir sobre situações, desenvolver soluções para a promoção da saúde pessoal e coletiva, e, consequentemente, aplicar os conhecimentos compreendidos (BRASIL, 1998).

O vídeo didático apresentado no início de cada atividade do Painel Itinerante, com abordagem conceitual de endoparasitos e ectoparasitos, foi uma estratégia de ensino importante, pois, apresentava os parasitos de maneira simples e didática. Desta forma, Coutinho (2009) ressalta que o filme coloca imagens e sons em movimento, faz aflorar as emoções, percebidas por meio dos sentidos, permite o conhecimento por meio do ponto de vista do outro, consequentemente o enriquecido de suas concepções.

Outra estratégia de ensino utilizada no Painel foi a demonstração de exemplares de parasitos fixados em meio líquido. Esta estratégia despertou a atenção 
dos estudantes, motivando-os e estimulando-os a participarem ativamente das aulas, tornando as aulas interativas e proporcionando a aprendizagem significativa. A motivação é fundamental nos processos de ensinar e aprender, Coll (1994) ressalta que os incentivos motivacionais são necessários na aprendizagem, pois permitem a aproximação dos conhecimentos prévios do estudante e com os conhecimentos científicos. Para Pozo (2002), a falta de motivação em sala de aula, pode provocar o fracasso na aprendizagem, assim, o conhecimento das condições que favorecem o processo de motivação de estudantes e professores é fundamental para o ensino.

Diante disso, ressalta-se o diferencial desta prática pedagógica, pois utilizava diversas estratégias de ensino, facilitando a aprendizagem, de forma prazerosa e relevante para os estudantes. Assim, inferimos sobre a importância de estudos dessa natureza, visto que, a parasitologia constitui uma prática de educação em saúde, sendo de fundamental relevância no bem-estar do indivíduo e na manutenção de um ambiente saudável. A implantação dessas atividades diferenciadas no ambiente escolar pode permitir ao estudante aplicar os conhecimentos teóricos e práticos à sua realidade social, bem como, o desenvolvimento de hábitos saudáveis. O projeto Painel Itinerante possibilitava os professores e os estudantes a identificarem as parasitoses de sua região, e compreender as profilaxias, os sintomas, a epidemiologia e o ciclo de vida. $\mathrm{Na}$ promoção de saúde é fundamental a atuação dos governantes, bem como, as medidas de educação preventiva, pois, como são as mais afetadas, as criança desempenham papel decisivo na prevenção das parasitoses.

De acordo com Toscani et al. (2007) a educação em saúde permite aos estudantes o desenvolvimento de hábitos saudáveis, para o seu bem estar e a manutenção de um ambiente saudável. Portanto, é fundamental que o processo educativo não ocorra de forma impositiva, mas adequada a suas capacidades cognitivas, num ambiente prazeroso, propiciando uma relação direta entre os conteúdos e o seu dia a dia. Entretanto, enfatizamos que as metodologias de aprendizagem não são perfeitas, pois os processos educativos são complexos, e envolvem vários fatores. De acordo com Pozo (2002, p. 249), “a aprendizagem é um problema complexo, [...] que não podem se identificar 'boas' e 'más' práticas didáticas, mas sim condições eficazes ou não para se alcançar os fins estabelecidos”. Para Coll e Solé (2004), a conexão de vários elementos, como: ensino do professor, aprendizagem do estudante, a interação entre professorestudante e estudante-estudante, conteúdos, dentre outros, compõem a essência dos processos educativos que precisam ser compreendidos e aperfeiçoados. 


\section{Considerações Finais}

O projeto Painel Itinerante - Parasitologia mostrou-se uma importante atividade na educação em saúde, pois a prática pedagógica diferenciada poderá influenciar no ambiente social dos estudantes, visto que estes poderão tornar-se divulgadores dos conhecimentos científicos.

Tendo em vista os aspectos observados, percebeu-se que as práticas pedagógicas tornam-se um diferencial nas aulas de ciências, principalmente no ensino de saúde, contrariando as aulas tradicionais utilizadas pela maioria dos professores. Deste modo, ressalta-se a importância das situações contexto, da problematização e interação no ensino de ciências, especialmente, no ensino de saúde para a promoção de aulas motivadoras e prazerosas, proporcionando a aprendizagem significativa, ou seja, a relação dos novos conhecimentos com os conhecimentos prévios dos estudantes.

As estratégias de ensino abordadas no Painel Itinerante como, o estudo de caso, o vídeo didático e os exemplares de parasitos despertaram a atenção dos estudantes e proporcionaram no ambiente escolar maior interesse pelos conteúdos abordados, pois permitiram relacionar a teoria com a prática, por meio dos processos interativos professor-estudante e estudante-estudante, promovendo assim, um espaço agradável e enriquecedor de aprendizagem.

Contudo, as metodologias perfeitas não existem, pois os processos de ensinar e aprender possuem grande complexidade. As estratégias de ensino somente auxiliam o professor, configuram-se como instrumentos para o ensino dos conteúdos e não como modelos a serem seguidos. Deste modo, cabe ao professor escolher a metodologia adequada para cada conteúdo, enfatizando o principal objetivo, a construção do conhecimento.

\section{Referências bibliográficas}

Asaolu, Samuel Oluwarotimi; Ofoezie, Ifeanyi Emmanuel. The role of health education and sanitation in the control of helminth infections. Acta Tropica, 86 (2), 283-294, 2003.

Brasil. Secretaria de Educação Fundamental, Ministério da Educação e do Desporto. Parâmetros curriculares nacionais: Temas Transversais - Saúde. Brasília, 1998a.

Brasil. Secretaria de Educação Fundamental, Ministério da Educação e do Desporto. Parâmetros curriculares nacionais: Ciências Naturais. Brasília, 1998b. 
Coll, César. Aprendizagem escolar e construção do conhecimento. Porto Alegre: Artes Médicas, 1994.

Coll, César; Solé, Isabel. Ensinar e Aprender no contexto da sala de aula. In: Coll, César; Marchesi, Alvaro; Palacios, Jésus. Desenvolvimento psicológico e educação: psicologia da educação escolar. 2. ed. (p. 242-260). Porto Alegre: Artmed, 2004.

Costa-Macedo, Leda Maria; Rey, Luis. Aleitamento e parasitismo infantil intestinal materno-infantil. Revista da Sociedade Brasileira de Medicina Tropical, 33 (4), 371$375,2000$.

Coutinho, Laura Maria. Cinema e educação: um espaço aberto. Salto para o futuro. TV Escola. Disponível em: < http://tvbrasil.org.br/fotos/salto/series/185114Cinemaeedu.pdf $>$ Acesso em 13 mai., 2012.

Delizoicov, Demetrio; Angotti, Jóse André; Pernambuco, Marta Maria. Ensino de ciências: fundamentos e métodos. São Paulo: Cortez, 2002.

Falcão, Jorge Tarcílioda Rocha; Ténier, Juan-Claude. Sobre os métodos quantitativos na pesquisa em ciências humanas: riscos e benefícios para o pesquisador. Revista Brasileira de Estudos Pedagógicos, 81 (198), 229-243, 2000.

Ferreira, Glauco Rogério; Andrade, Carlos Fernando Salgueirosa. Alguns aspectos socioeconômicos relacionados a parasitoses intestinais e avaliação de um intervenção educativa em escolares de Estiva Gerbi, SP. Revista da Sociedade Brasileiro de Medicina Tropical, 38 (5), 402-405, 2005.

Ikegami, Marcelo Tetsuo; Coelho, Lina Maria de Petrini da Silva; Aidar Sobrinho, Tuffi; Oliveira, Sônia Maria; Yoshizumi, Alexandre Massao; Nakamoto, Allyson Yukio Koda; Brotto, Sérgio de Arruda, Felberg, Sergio, \& Maiorano, Marcelo Raymundo. Ovos e larvas de helmintos nos sanitários de pré-escolas municipais de Sorocaba, SP e suas freqüências nas fezes das crianças. Revista da Sociedade Brasileira de Medicina Tropical, 32 (6), 647-652, 1999.

Pedroso, Reginaldo; Siqueira, Rosângela. Pesquisa de cisto de protozoários e larvas de helmintos em chupetas. Jornal de Pediatria, 73 (1), 21-25, 1997.

Pozo, Juan Ignacio. Aprendizes e mestres: a nova cultura da aprendizagem. Porto Alegre: Artmed, 2002.

Santa Catarina. Secretaria de Estado da Educação. Proposta Curricular de Santa Catarina. Florianópolis. Disponível em: < http://www.sed.sc.gov.br/>. Acesso em: 13 mai. 2012.

Toscani, Nadima Vieira; Santos, Antônio José Duarte Silva; Silva, Leonardo Leiria de Moura da; Tonial, Cristian Tedesco; Chazan, Marcio; Wiebbelling, Adília Maria Pereira; Mezzari, Adelina. Development and analysis of an educational game for children aiming prevention of parasitological diseases. Interface, 11 (22), 281-94, 2007. 
Vasconcellos, Celso dos Santos. Construção do Conhecimento em sala de aula. São Paulo: Libertad, 2005.

Zaiden, Marilúcia Fonsec; Santos, Branca Maria de Oliveira; Cano, Maria Aparecida Tedeschi; Nascif Júnior, Iucuf Abrão. Epidemiologia das parasitoses intestinais em crianças de creches de Rio Verde - GO. Medicina (Ribeirão Preto), 41 (2), 182-187, 2008 . 